\title{
External Inversion of Thin-walled Corrugated Tubes
}

\author{
Yang $\mathrm{Li}^{1}$ and Zhong $\mathrm{You}^{2, *}$
}

1 Graduate Aerospace Laboratories, California Institute of Technology, 1200 E. California Blvd., Pasadena CA 91125, USA

${ }^{2}$ Department of Engineering Science, University of Oxford, Parks Road, Oxford, OX1 3PJ, U. K.

*Corresponding author. Email: zhong.you@eng.ox.ac.uk

\begin{abstract}
A thin-walled circular tube is known to be able to invert externally under axial compression when it is placed upon a profiled rigid die. Such a structure has become a promising candidate for energy absorbing applications due to its high reaction force and good load uniformity. However, the inversion process could become rather unstable when imperfections are present and the friction between the tube and die is high, which largely limits its application. In this paper we propose the use of corrugated thin-walled tubes. Both simulation and experimental results show that thin-walled corrugated tubes can have much more stable inversion in comparison with their circular counterparts. They are less sensitive to the surface imperfections, and no stringent requirement of lubrication on the die is necessary. Our research has paved the way for such devices to be used as an effective energy absorption device in many practical applications.
\end{abstract}

keywords: external inversion, thin-walled tube, axial compression, corrugation 


\section{Introduction}

Thin-walled tube inversion is a classic problem in the research of thin-walled structures. It is a process where a tube under compression undergoes an inside-out roll-up with the help of a profiled die. This process can be effectively utilised for energy dissipation.

Extensive studies were performed on tube inversion [1-6]. The tube can invert inwards or outwards. The latter is known as the external inversion [1]. which takes place with the assistance of a rigid die placed at one end of the tube when the tube is subjected to axial compression. A schematic diagram of this process is shown in Fig. 1. Successful tube inversion is subjected to a number of stringent conditions. Rosa summarised four controlling factors that govern the inversion of a tube as $D / t, r_{\text {die }} / R$, $\mu$, and strain hardening exponent, where $D$ is the diameter of the tube, $t$ is its wall thickness, $r_{\text {die }}$ is the die radius, $R$ is tube's cross-section radius, and $\mu$ is friction coefficient between the tube and the die [2]. Failure of selecting appropriate values for those parameters results in either fracturing or buckling of the tube other than inverting. Avoidance of fracturing is straight forward, which requires smaller die radius to reduce the amount of circumferential stretch and the use of ductile materials capable of larger strain. However, eliminating the occurrence of buckling requires careful assignment of all four parameters, which is essentially to ensure that the buckling load of the tube is higher than the load required for inversion, so that inversion occurs instead of buckling.

All successful inversion adopted some sort of lubrication, which can effectively reduce the load needed for inversion to a lower value than its buckling load. Without a proper lubrication, $\mu$ would increase, e.g., from 0.02 to 0.4 . Local buckling would easily take place $[2,3]$.

It has also been noticed that the inverting load drops faster than the buckling force with an increase of $D / t$ ratio [4], that would make the occurrence of inversion easier and more reliable albeit at the cost of a reduced specific energy absorption capacity (SEA). Larger $D / t$ ratio also allows smaller $r_{\text {die }} / R$ [4]. Tube inversion with smaller $r$ die/ $R$ is less likely to fracture [3]. These give a good reason to favour tubes with larger $D / t$ ratio in order to achieve inversion.

A further factor to consider is the imperfection within thin-walled tubes. It is known that thinner circular tubes are more sensitive to imperfection $[7,8]$, and the presence of imperfection considerably reduces the actual buckling force of the tube. As a result, imperfections increase the likelihood of tube to buckle. It was shown previously that a way to increase the buckling load of a thin-walled tube is to introduce corrugation to the cross-section of a tube [9]. The presence of corrugation also reduces the imperfection-sensitivity. In consequence of those merits, we envisage that a corrugated tube with a 
larger $D / t$ ratio may have a stable and more consistent inversion. Our investigation is presented in this paper.

The layout of this paper is as follows. Section 2 contains the numerical simulation of thin-walled circular tube inversion with $D / t \geq 100$. The results of the analysis are used for comparison with those of their corrugated counterparts. Section 3 introduces the design of thin-walled corrugated tubes, followed by a detailed analysis of these tubes without and with imperfections. The experiments of thinwalled corrugated tube inversion are given in Section 4. A parametric study is presented in Section 5, and finally, discussions in Section 6 concludes the paper.

\section{Behaviour of Thin-walled Circular Tubes under Compression}

Consider a set of thin-walled circular tubes made from 304 stainless steel, which has density $\rho=$ $8030 \mathrm{~kg} / \mathrm{m}^{3}$, Young's Modulus $E=193 \mathrm{GPa}$, Poisson's ratio $v=0.3$, yield stress $\sigma_{\mathrm{y}}=241.3 \mathrm{MPa}$, ultimate stress and strain $\sigma_{\mathrm{u}}=679.6 \mathrm{MPa}, \varepsilon_{\mathrm{u}}=55.8 \%$, and strain hardening formula $\sigma=1400 \varepsilon^{0.43}$. The tubes have a diameter $D=50 \mathrm{~mm}$ and length $L=100 \mathrm{~mm}$. The thicknesses are set as $0.5 \mathrm{~mm}, 0.35$ $\mathrm{mm}$, and $0.25 \mathrm{~mm}$, leading to $D / t \geq 100$. A $20 \mathrm{~mm}$ tall rigid die is used with $r_{\text {die }}=4 \mathrm{~mm}$. The reason for choosing such a die radius is due to the following reasons. First, if $r_{\mathrm{die}}<4 \mathrm{~mm}$ (with $D=50 \mathrm{~mm}$ ), it would make the leading edge of inverted part be pressed against the tube and thus cause local buckling as pointed out by Reid [1]. Secondly, a larger die radius could generally increase the difficulty of tube inversion due to an increase in circumferential stretch. We assumed a case where no superficial lubrication is applied. The friction coefficient is set as $\mu=0.32$ (This is validated by the experiments outlined in Section 4).

Abaqus Explicit Dynamic and general contact with penalty method for friction were used for simulation [10]. After convergence tests, a total of 15700 S4R elements were used for meshing each of the tubes. The two sets of simulations were performed. Tubes were placed between two rigid plates for the buckling analysis, whereas in the inversion analysis, a rigid die replaced the bottom plate which was modelled with 8753 R3D4 and 149 R3D3 elements. The simulations were controlled by the displacement between the rigid ends. We superficially ramped up the loading speed to $0.8 \mathrm{~m} / \mathrm{s}$ for inversion and $0.05 \mathrm{~m} / \mathrm{s}$ for buckling to accelerate the computation. These speeds were adequate as the kinematic energies were well below $5 \%$ so that the simulations could be regarded as quasi-static. At these loading speeds, the inertial force does not change the mode of deformation. 
Results of simulations are presented in Table 1 , which shows that only tubes with $t=0.35 \mathrm{~mm}$ and $0.25 \mathrm{~mm}$ were inverted successfully. As expected, ratios between the buckling and inverting forces indicate that a successful inversion requires them to be considerably larger than 1 . Note that both forces are converted to forces per unit area by taking into account of the cross-sectional areas of the material, which is necessary for a meaningful comparison with the results obtained for the corrugated tubes later.

For $t=0.5 \mathrm{~mm}$, an obvious buckling mode was observed in buckling analysis when the displacement of top rigid plate reached $2.5 \mathrm{~mm}$, Fig. 2(a). In the inversion analysis, the tube was initially inverted until the displacement of the top plate reached $19.2 \mathrm{~mm}$, and then it buckled in an axisymmetric mode, Fig. 2(b). Reactions of both buckling and inverting process vs. axial displacement were plotted in Fig. 2(c), where the reaction is the total reaction force divided by the cross-section area, and the buckling and inversion curves are plotted with the displacement scales placed on the top and at bottom of the diagram, respectively (The same applies to all the subsequent reaction force vs. displacement diagrams). From the plot it can be seen that, when the reaction of inversion reaches the buckling force, the tube starts to buckle instead of continuing the inversion process. In consequence, the reaction force drops down quickly.

Figures 3(a) and (b) show inversion process of the tube with $t=0.25 \mathrm{~mm}$. The corresponding reaction force vs. displacement curves are given in Fig. 3(c), which also contains the curve when the tube is buckled under compression. The buckling force, which is the peak in the buckling analysis curve, is about $22 \%$ greater than the inversion force, the average force when steady inversion takes place. The ratio between the buckling force and the inversion force is 1.22 , see Table 1.

The inversion process of tube with $t=0.35 \mathrm{~mm}$ is similar to that of tube with $t=0.25 \mathrm{~mm}$. The specific energy absorption (SEA) of both inverted tubes are given in Table 1.

Imperfections influence the behaviour of the tubes significantly, especially when the tubes are thin. Consider the buckling and inverting performances of imperfect tubes with $t=0.25$ and $0.35 \mathrm{~mm}$, respectively, where the imperfect shape is chosen as the first buckling mode with an amplitude of $0.1 \mathrm{~mm}$. Since the imperfection amplitude and its distribution cannot be measured accurately, this value was chosen so that simulations best match with experiments (shown in Section 4). This value is only applicable to tubes with thickness between 0.25 and $0.35 \mathrm{~mm}$. As expected, the buckling forces for both tubes are reduced because of the imperfections, see Table 2. The reduction is more significant for the thinner tube. The simulation showed that in both cases, a very small amount of inversion was possible, but it soon was over-ridden by the buckling mode. Figures 4(a) and (b) display the 
deformation process of the imperfect tube with $t=0.25 \mathrm{~mm}$. The reaction force vs. displacement curves for the tube with $t=0.25 \mathrm{~mm}$ is given in Fig. 4(c). In both tubes, the drop in the buckling forces due to imperfections makes them rather close to the inversion forces, which makes inversion impossible.

In summary, the sensitivity of a very thin tubes to imperfection largely lowers their actual buckling force, resulting in that tubes fail to be inverted with imperfect surface geometry, especially with a frictional contact $(\mu=0.32)$. Further measures are necessary to overcome this problem, which has led to our proposal to use corrugated tubes outlined next.

\section{Corrugated Tube and Its Inversion}

\subsection{Design}

Figure 5(a) is a corrugated tube whose cross-section is shown in Fig. 5(b). $R_{\mathrm{i}}, \alpha R_{\mathrm{i}}$ and $\theta$ are the inner radius of the tube, the amplitude of corrugation and the central angle corresponding to each corrugation, respectively. $\theta=180^{\circ} / n$ where $n$ is the number of corrugation pairs. The shape geometry of cross-section is therefore determined by three independent parameters: $R_{\mathrm{i}}, \alpha$ and $\theta$. The corrugation is made from circular arcs of identical radius, i.e., $r_{1}=r_{2} . \theta_{1}$ and $\theta_{2}$ are the corresponding central angles

of the circular arcs, respectively, whereas $R_{\mathrm{m}}$ and $R_{\mathrm{o}}$ are the mid and outer radii of the tube, respectively. Note that $R_{\mathrm{m}}$ is not halfway between $R_{\mathrm{i}}$ and $R_{\mathrm{o}}$; rather it is the radius of the turning point of inner and outer corrugations. Geometrically, there are

$$
r_{1}=r_{2}=\frac{R_{\mathrm{i}}\left[\alpha+\alpha \tan ^{2}\left(\frac{\theta}{2}\right)+4 \tan ^{2}\left(\frac{\theta}{2}\right)\right]}{4}+\frac{R_{\mathrm{i}} \tan ^{2}\left(\frac{\theta}{2}\right)}{\alpha}
$$

and

$$
\theta_{1}=\theta_{2}=2 \arcsin \left[\frac{\alpha^{2} \sin (\theta)+2 \alpha \sin (\theta)}{2 \alpha-2 \cos (\theta)-2 \alpha \cos (\theta)+\alpha^{2}+2}\right]
$$

In the subsequent analysis and experiments, we choose $R_{\mathrm{i}}=25 \mathrm{~mm}, \alpha=0.04, \theta=5^{\circ}(n=36)$ and the length of the tube $L=100 \mathrm{~mm}$.

\subsection{Tubes Buckling and Inversion}


The corrugated tube is made from the same material as for circular tubes presented in Section 2. Again we consider three tubes with thickness $t$ set as $0.5 \mathrm{~mm}, 0.35 \mathrm{~mm}$ and $0.25 \mathrm{~mm}$, respectively. For inversion, a rigid die is used with the friction coefficient between the die and the tube $\mu=0.32$. The profile of the die, shown in Fig. 5(c), is identical to that used for circular tube inversion. Die radius $r_{\mathrm{die}}=6 \mathrm{~mm}$, which differs from die radius of circular tube because a larger circumferential expansion is required for corrugated tubes. The selected $r_{\text {die }}$ enables the corrugation to be completely stretched before inversion takes place. The height of the rigid die remains $20 \mathrm{~mm}$.

The numerical simulation was conducted using Abaqus Explicit [10], with displacement controlled loading. The model consisted of 72000 S4R elements for the tube, whereas the die was set as rigid body modelled by R3D4 and R3D3 elements as in the simulation of circular tubes.

All of the tubes were inverted successfully. The buckling and inversion forces and SEA are given in Table 3. It can be seen that the SEA of both tubes are less than those of their circular counterparts. This is understandable because the corrugation requires more materials since we kept $R_{i}$ the same as the radius of the circular tubes.

The corrugated tube with $t=0.5 \mathrm{~mm}$ was successfully inverted as shown in Fig. 6(a). Through buckling and inverting analysis, respectively, the reaction force vs. displacement curves were obtained, Fig. 6(b). Evidently the buckling load is higher than the load needed for inverting. The ratio between the two is 1.26, which allows stable inversion instead of buckling. Both corrugated tubes with $0.35 \mathrm{~mm}$ and $0.25 \mathrm{~mm}$ thickness were inverted successfully, too, and the reactions force vs. displacement curves are shown in Fig. 7(a) and (b), respectively.

\subsection{Imperfect Corrugated Tubes Buckling and Inversion}

Similar to the last section, imperfect shapes were generated by the superposition of perfect shape and its first buckling mode with an amplitude of $0.1 \mathrm{~mm}$. Imperfect corrugated tubes with $0.5 \mathrm{~mm}, 0.35$ $\mathrm{mm}, 0.25 \mathrm{~mm}$ thicknesses were subjected to compression without and with a die for buckling and inverting analysis, respectively.

All three tubes were inverted successfully, and results are presented in Table 4. Figure 8 shows the partially inverted tubes and buckling and inverting reaction force vs. the crushing displacement curves of the three tubes are displayed in Fig. 9. 
Comparing the performances between the perfect and imperfect corrugated tubes, it becomes apparent that the presence of imperfection reduces the buckling forces (the third columns of Table 3 and 4) but the inversion forces of the imperfect corrugated tubes hardly change (the fourth columns of Tables 3 and 4). This suggests that the imperfection has little or no influence on the inversion force of a corrugated tube.

\subsection{Comparison between circular and corrugated tubes}

A few observations can be made based on the simulation results. First, after the introduction of the corrugation, the tube with $t=0.5 \mathrm{~mm}$ becomes invertible even with imperfection. For the other two thinner tubes, we group together the key data from simulations in Table 5 in order to facilitate the comparison. For the perfect tubes, the introduction of corrugation slightly increases the buckling forces (the second columns in Table 5), but it significantly reduces the force needed for inversion (the third columns of Table 5). This is due to the fact that the corrugation makes circumferential stretch, a process required to enable inversion, easier. When imperfection is present, the buckling forces for circular tubes are reduced, making them impossible to invert. The same reduction in the buckling forces is recorded for the thin corrugated tubes, but our numerical simulation demonstrates that they can still be inverted stably with frictional contact. This is, however, at the cost of a decrease in SEA when compared with those of the perfect circular counterparts. It can be seen that the SEAs for both $0.35 \mathrm{~mm}$ and $0.25 \mathrm{~mm}$ thick corrugated tubes are $22.7 \mathrm{~kJ} / \mathrm{kg}$ and $19.6 \mathrm{~kJ} / \mathrm{kg}$ (the sixth column of Table 5), whereas those of the perfect circular tubes of same thickness are $27.6 \mathrm{~kJ} / \mathrm{kg}$ and $25.2 \mathrm{~kJ} / \mathrm{kg}$, giving a reduction of $17.8 \%$ and $22.2 \%$, respectively.

\section{Experiments of Circular and Corrugated Tubes Inversion}

In this section, circular and corrugated tubes inversion were experimentally tested. On the other hand, numerical and theoretical models from previous sections were compared and verified by experimental results.

\subsection{Specimens and Experimental Settings}

A set of experimental specimens were manufactured from solid 304 stainless steel rod by a wire electrical discharging machine. The specimens included a circular tube Cir1, and three corrugated tubes, Cor1, Cor2 and Cor3 with $t=0.25$, shown in Fig 10(a). Also in display are two dies, Die4 with $r_{d i e}=4 \mathrm{~mm}$ and Die6 with $r_{d i e}=6 \mathrm{~mm}$, made from Carrs P552. Instron 5982 was used for compression tests, and setting of tests is shown in Fig. 10(b). Speed of loading was at $10 \mathrm{~mm} / \mathrm{min}$, and no lubrication 
was used for the contact. The geometries of these specimens were same with those given in Sections 2 and 3, and all their wall thicknesses were intended for $0.25 \mathrm{~mm}$. However, due to a small manufacturing inaccuracy, the average thickness of Cor 2 and Cor3 were found to be $0.29 \mathrm{~mm}$.

\subsection{Experimental Results and Comparison with Numerical Simulations}

The numerical simulations were performed with a slight adjustment to account for the wall thickness variation. $\mu$ was taken as 0.32 . The surface imperfection amplitude was $0.1 \mathrm{~mm}$ as in the previous analysis. Since the direct measurements of both properties were difficult, the two values were obtained by fitting the numerical results to the experimental data, as it was done in [11]. Note that both values fall into a reasonable range provided by $[2,3,9]$. The simulation results are then used for comparison with experimental measurements.

For the circular tube Cir 1 both experimental and the corresponding numerical results are given in Fig. 11. The tube failed to invert. In the experiment, the circular tube developed axisymmetric concertina folding at first with the lateral support from the die, and then quickly switched to nonaxisymmetric mode. In simulation, the tube failed only in non-axisymmetric mode. This discrepancy is reflected in the plots shown in Fig. 11(c) where the experimental result has three obvious peak values whilst the numerical result has only one. The buckling forces of both results are almost identical, which suggests that $0.1 \mathrm{~mm}$ amplitude for imperfection was reasonable.

Results of the corrugated tubes with $t=0.25 \mathrm{~mm}$ (Cor 1) and $0.29 \mathrm{~mm}$ (Cors 2 and 3 ) are presented in Figs. 12 and 13, respectively. All the corrugated tubes were successfully inverted. Good consistency between experimental and numerical results was observed in terms of both deformed shapes and reaction forces vs. displacement curves. This validates our choice of $\mu$.

\subsection{Discussions}

We experimentally demonstrated that thin corrugated tubes could be inverted reliably without any lubrication. The good consistency between the numerical and experimental results prove that our simulations were accurate in predicting the inversion behaviour of thin-walled tubes with imperfection.

Note that in Fig. 13(c), reaction force of Cor2 gradually rose up after reaching a displacement of $30 \mathrm{~mm}$, which was due to its self-contact between inverted outer tube and inner tube at two sides, shown in Fig.14.

\section{Parametric Study}


The correlation between the simulations and experiments prompted us to perform a parametric study to examine whether corrugated tubes with other geometrical parameters and thickness could be also inverted. By altering $\theta$ and $\alpha$, a set of different corrugated tubes were generated, see Fig. 15(a) and (b). All tubes have length of $100 \mathrm{~mm}, R_{i}$ of $25 \mathrm{~mm}$, and are made from the same material as those presented in the previous sections. No imperfection was considered here since we have shown that the corrugated tubes are insensitive to imperfection. $\mu$ was raised to 0.4 (from 0.32 ) to conservatively ensure that the tubes can invert without lubrication.

Simulation results with corresponding tubes are given in Table 6. It can be observed that the failure modes can be categorised as inversion and buckling. Both inversion and buckling failures have been extensively discussed in Section 3. The ratios between buckling and inversion forces are given under the column marked as force ratio in the table. It shows that higher $\alpha$ and smaller $\theta$ give a higher ratio between buckling and inverting force, which stands for better reliability of achieving successful tube inversion. Note that the corrugated tubes are heavier than their circular counterparts of the same diameter and thickness. Although they have more reliable inversion, the inversion process involves less circumferential stretching because of corrugation. Both factors result in lower SEAs than those of the circular counterparts. Hence, for any practical application, the optimum design should be the tube with the smallest $\alpha$ (corrugation amplitude) that can have a successful inversion whilst maintaining a decent SEA.

Based on the analysis, a preferable range of parameters is given in Table 7, which can be used as a design guide.

\section{Conclusion}

This paper has presented several findings relating to external inversion of thin-walled tubes. Through both numerical simulations and experiments, we have shown that though thinner circular tubes are more likely to be inverted in theory, they are very sensitive to surface imperfection. Moreover, a successful inversion requires lubrication between the tubes and the dies. These issues largely reduce the possibility of a successful inversion, making them ill-suited as an energy absorption device in practice. The introduction of corrugation into cross-section not only increases the practical buckling force, but also decreases the load (per area) needed for inversion due to the fact that it becomes easier to circumferentially stretch the tube during inversion. Most importantly, the corrugated tubes are far less sensitive to surface imperfections, and require no lubrication between the tube and die. In 
consequence, thin-walled corrugated tube can be inverted reliably. Our findings have greatly extended the utilisation of the inversion mechanism in practical applications.

Though all of our prototypes were made by cutting solid 304 stainless steel rod with a wire electrical discharging machine to reduce imperfections, this technique can be rather expensive in practice. However, with modern extrusion technique, the corrugated tubes can be extruded relatively easily at low cost just like many other sectional profiles commercially available. Alternatively, one may roll a circular tube over a pair of moulds with corrugation, or make a corrugated sheet and then weld two sides together.

For circular tube inversion, a few theoretical models have been proposed to predict the inversion forces $[1,12]$. These models, however, are restricted to the cases where the frictions between the tube and die is either very small or zero. Following this paper, it is worthy of further investigation to develop models taking into account the unlubricated contact between the tube and die. Moreover, we are investigating the possibility of developing a theoretical model to estimate the inversion force for the corrugated tubes. We shall report our findings in due course.

\section{Acknowledgement}

The authors wish to acknowledge the support of Air Force Office of Scientific Research (FA9550-161-0339).

\section{References}

1. Reid, S.R., Plastic deformation mechanisms in axially compressed metal tubes used as impact energy absorbers. International Journal of Mechanical Sciences, 1993. 35(12): p. 1035-1052.

2. Rosa, P.A., et al., An investigation on the external inversion of thin-walled tubes using a die. International Journal of Plasticity, 2004. 20(10): p. 1931-1946.

3. Sekhon, G.S., N.K. Gupta, and P.K. Gupta, An analysis of external inversion of round tubes. Journal of Materials Processing Technology, 2003. 133(3): p. 243-256.

4. El-Domiaty, A., Curling of thin tubes: Analytical and experimental study. Journal of Materials Engineering and Performance, 1997. 6(4): p. 481-495.

5. P. K. Gupta, N.K.G., Investigation of External Inversion of Thin-Walled Tubes. 2013: Proc. Indian Natn. Sci. Acad. 79 No. 4.

6. Lu, G. and T.X. Yu, Energy Absorption of Structures and Materials. 2003: Woodhead Publishing.

7. Brush, D.O. and B.O. Almroth, Buckling of bars, plates, and shells. 1975: McGraw-Hill.

8. Jones, R.M., Buckling of Bars, Plates, and Shells. 2006: Bull Ridge Publishing. 
9. Ning, X. and S. Pellegrino, Imperfection-Insensitive Axially Loaded Cylindrical Shells. 54rd AIAA/ASME/ASCE/AHS/ASC Structures, Structural Dynamics and Materials Conference, 2013.

10. Dassault Systems SIMULIA corp., Abaqus Documentation Version 6.13, Providence, RI, USA, 2013.

11. S.R. Reid, J.J. Harrigan, Transient effects in the quasi-static and dynamic internal inversion and nosing of metal tubes, International Journal of Mechanical Sciences, 1998. 40(2): p. 263-280.

12. X. Yu, X. Qiu, T. Yu, Theoretical model of a metal tube under inversion over circular dies, International Journal of Mechanical Sciences, 2016, 108: p. 23-28. 


\section{External Inversion of Thin-walled Corrugated Tubes}

Figures:

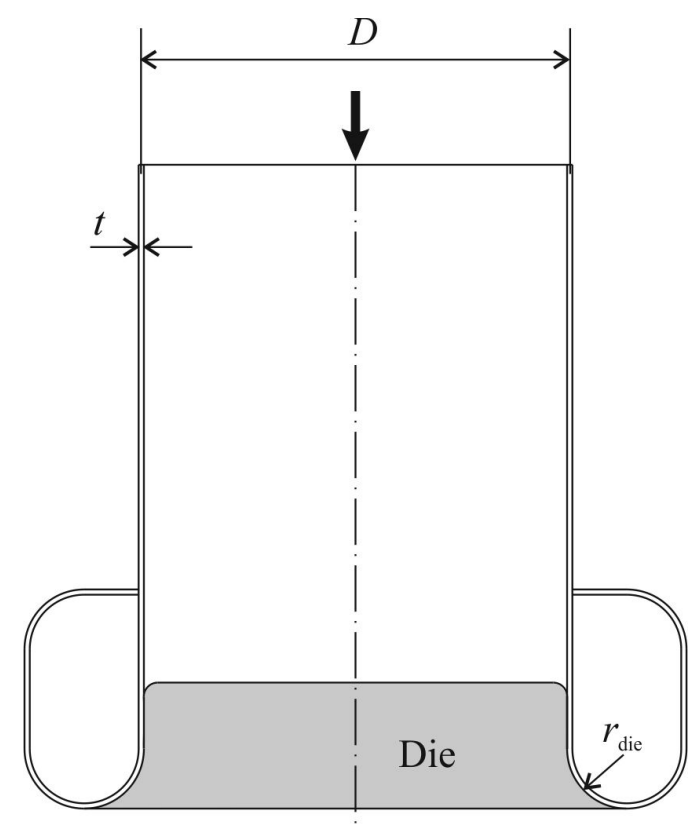

Figure 1. External inversion of a thin-walled circular tube. 


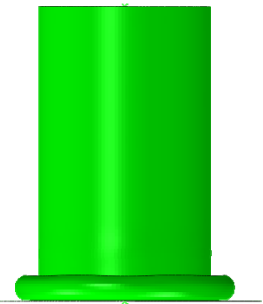

(a)

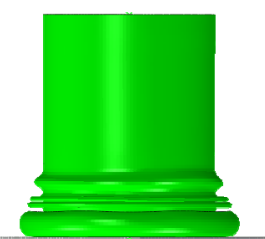

(b)

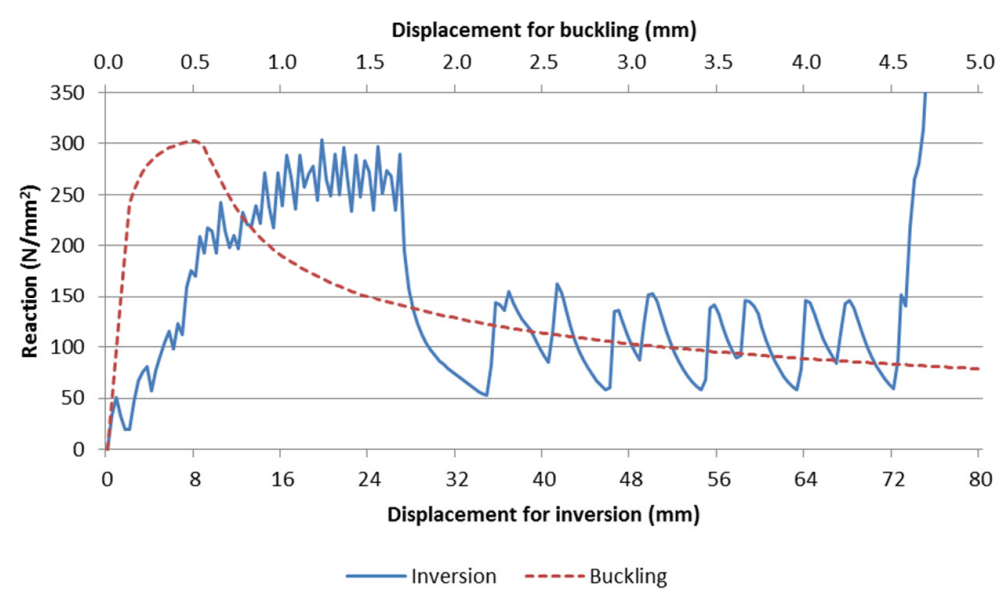

(c)

Figure 2. Buckling and inverting of tube with $D=50 \mathrm{~mm}, L=100 \mathrm{~mm}$ and $t=0.5 \mathrm{~mm}$. (a) Buckling analysis when shortening reached $2.5 \mathrm{~mm}$; (b) inverting analysis showing that inversion took place when shortening reached $19.2 \mathrm{~mm}$ and $40 \mathrm{~mm}$, respectively; and (c) reaction force vs. displacement curves. 


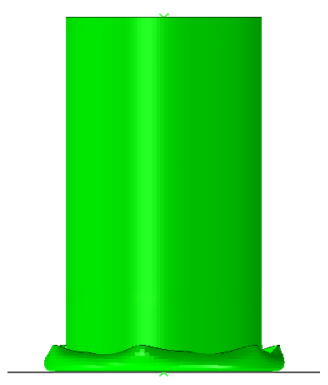

(a)

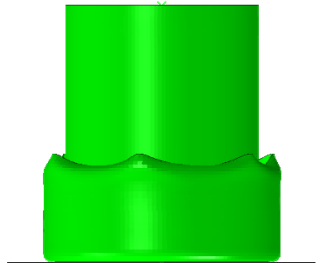

(b)

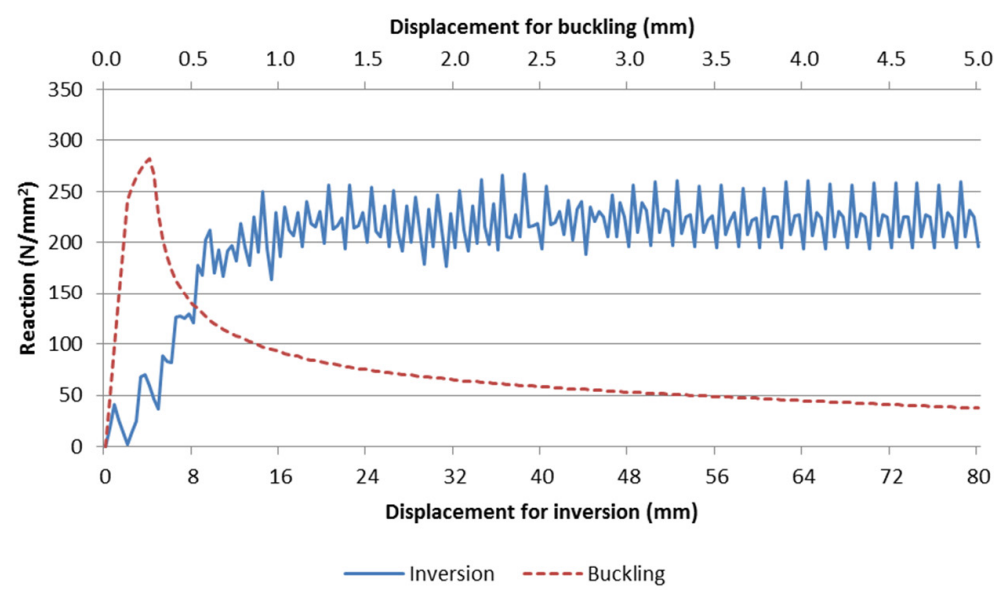

(c)

Figure 3. Buckling and inverting of tube with $D=50 \mathrm{~mm}, L=100 \mathrm{~mm}$ and $t=0.25 \mathrm{~mm}$. (a) Buckling analysis when shortening reached $2.5 \mathrm{~mm}$; (b) inverting analysis when shortening reached $16 \mathrm{~mm}$ and $40 \mathrm{~mm}$, respectively; and (c) reaction force vs. displacement curves. 


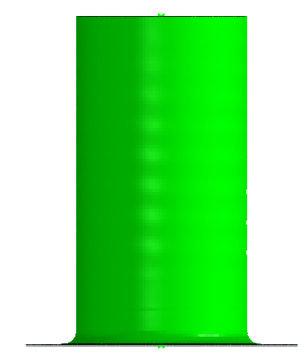

(a)

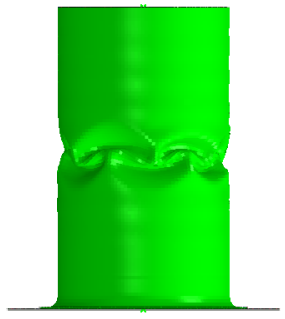

(b)

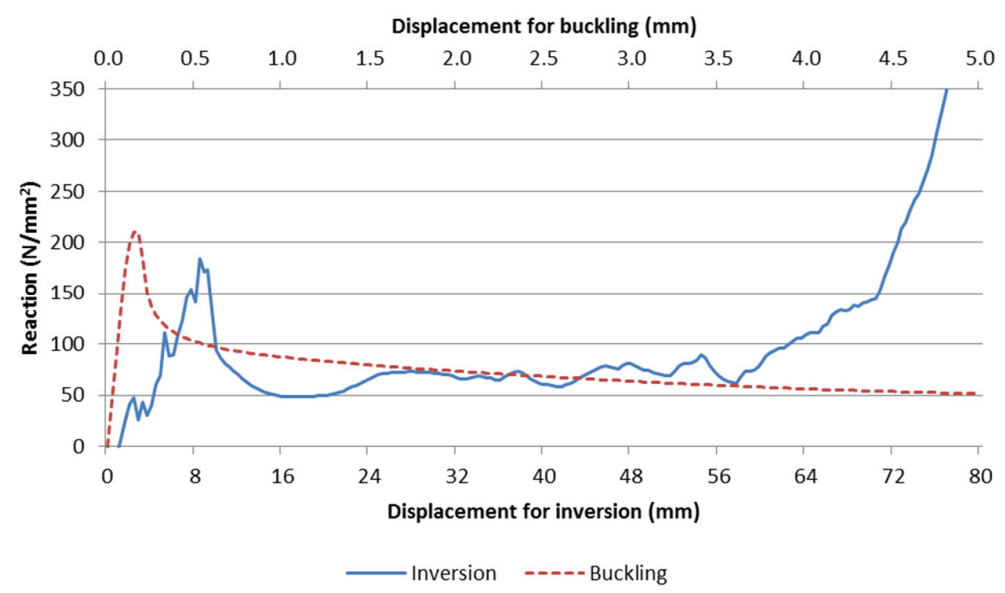

(c)

Figure 4. Buckling and inverting of imperfect tube with $D=50 \mathrm{~mm}, L=100 \mathrm{~mm}$ and $t=0.25 \mathrm{~mm}$. (a) Buckling analysis when shortening reached $2.5 \mathrm{~mm}$; (b) inverting analysis when shortening reached $8 \mathrm{~mm}$ and $16 \mathrm{~mm}$, respectively; (c) reaction vs. displacement curves. 


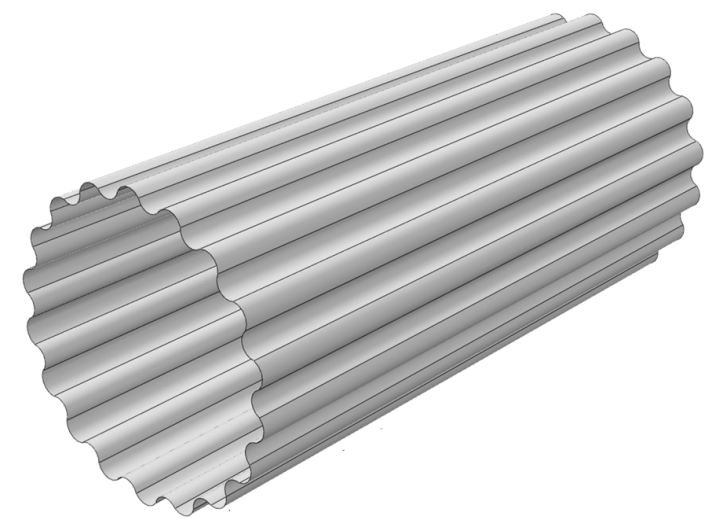

(a)

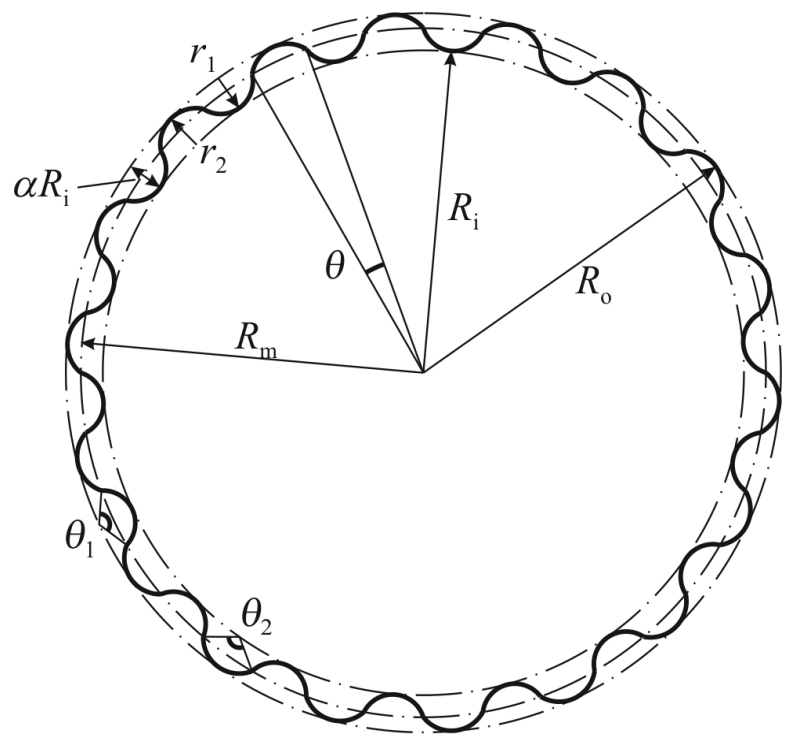

(b)

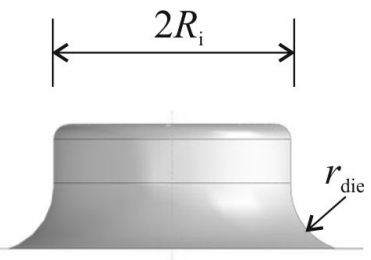

(c)

Figure 5. Design of corrugated tube and its die. (a) 3D shape of a corrugated tube; (b) its cross section; and (c) the die used for external inversion. 


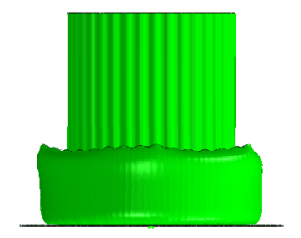

(a)

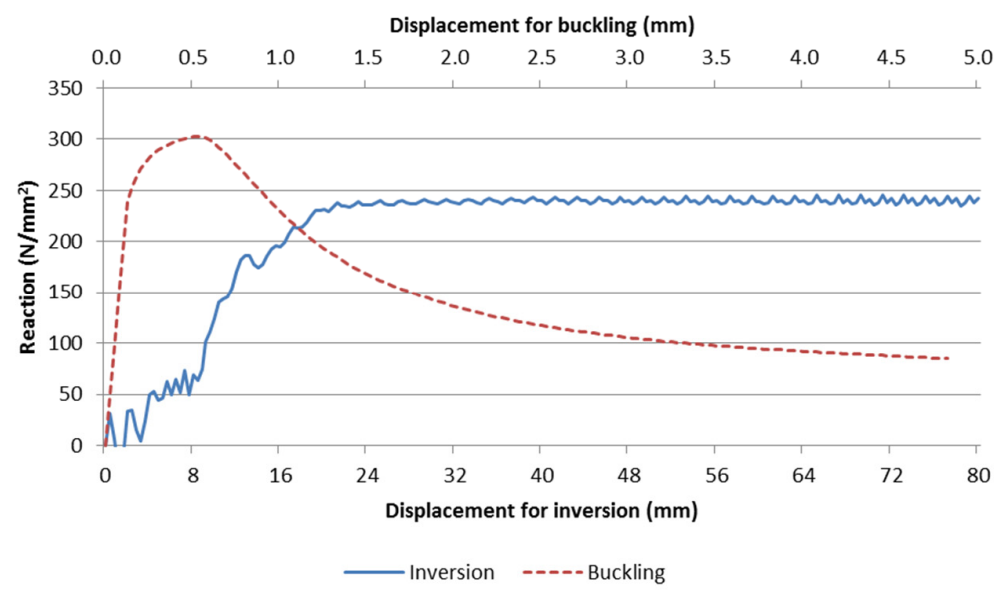

(c)

Figure 6. (a) Inversion of a corrugated tube with $D=50 \mathrm{~mm}, L=100 \mathrm{~mm}$ and $t=0.5 \mathrm{~mm}$; (b) reactions vs. displacement curves when the tube is subjected to buckling and inverting, respectively. 


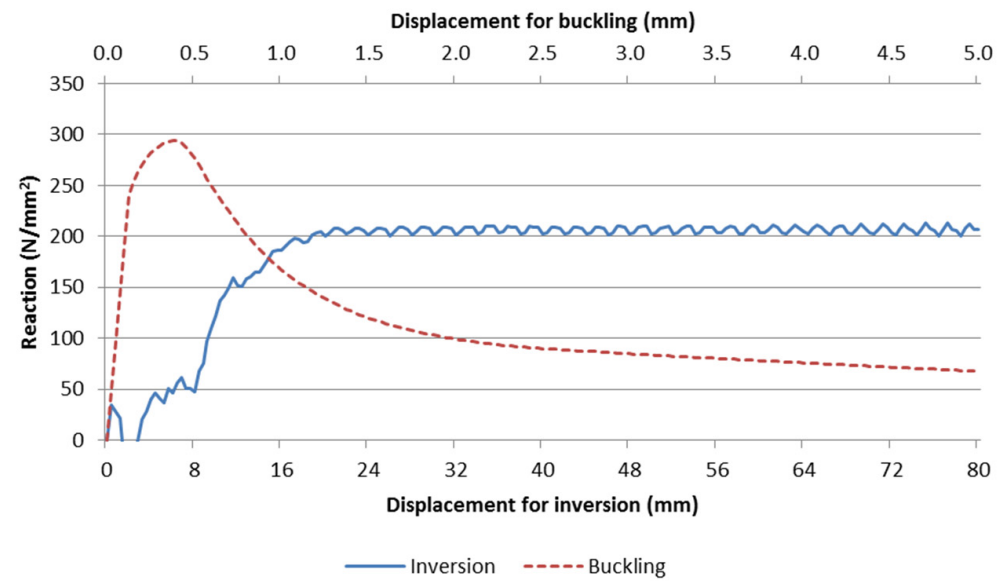

(a)

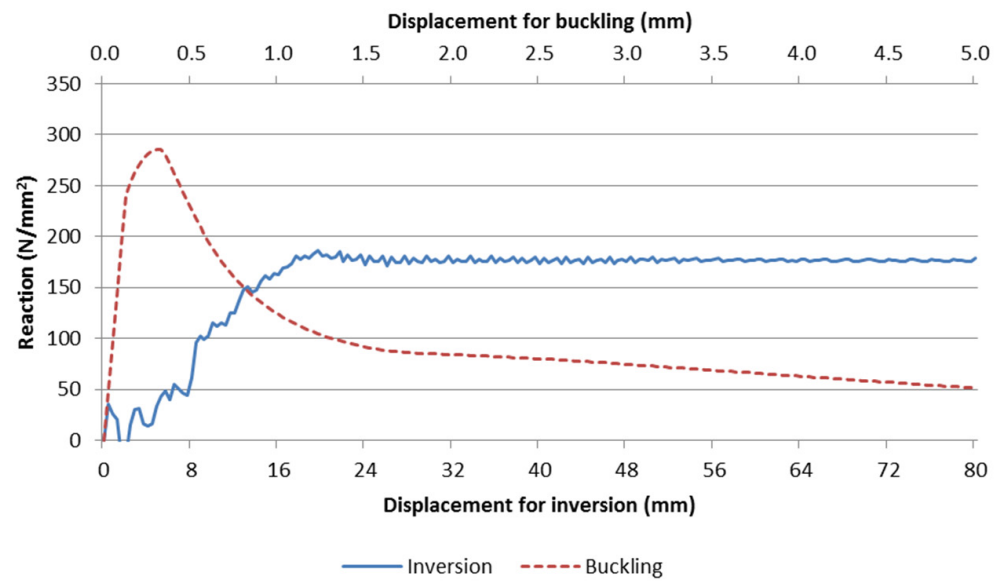

(b)

Figure 7. Reactions vs. displacement curves for corrugated tubes with (a) $t=0.35 \mathrm{~mm}$ and (b) $t=$ $0.25 \mathrm{~mm}$. 


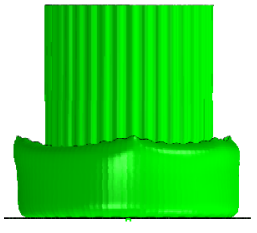

(a)

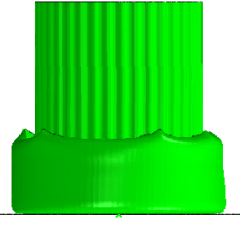

(b)

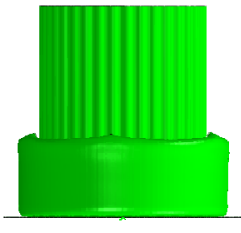

(c)

Figure 8. Partially inverted imperfect corrugated tubes with (a) $t=0.5 \mathrm{~mm}$, (b) $t=0.35 \mathrm{~mm}$ and (c) $t=0.25 \mathrm{~mm}$. 


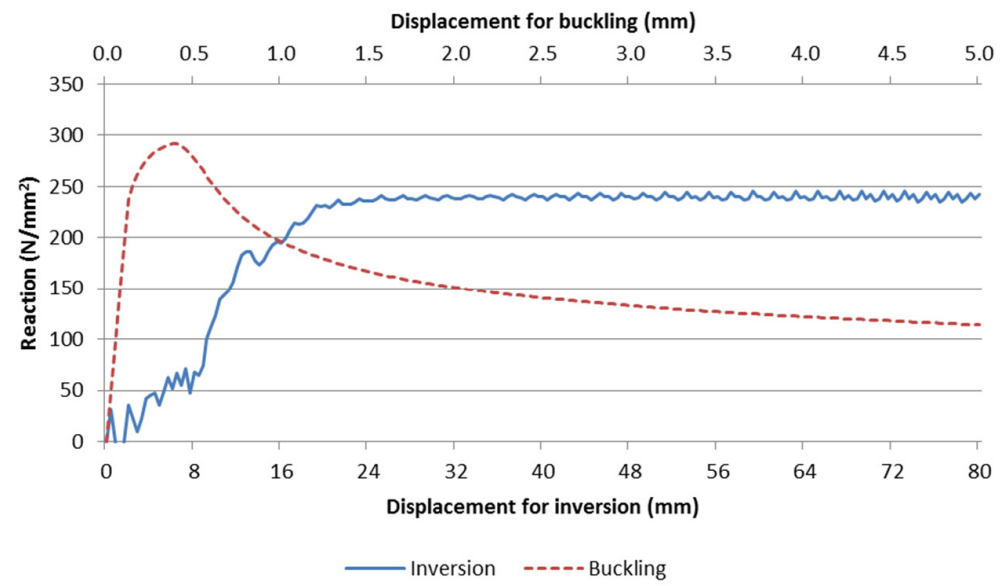

(a)

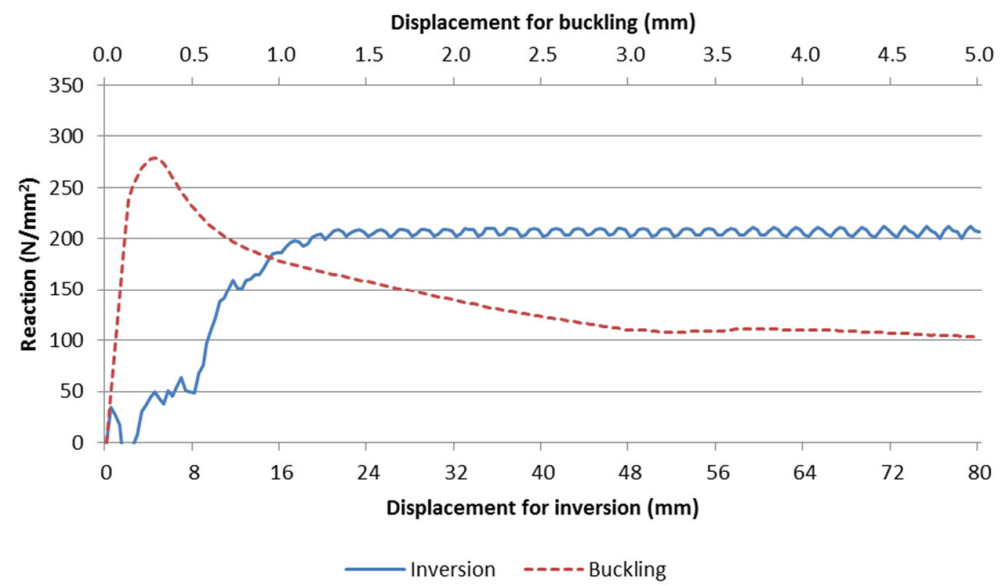

(b)

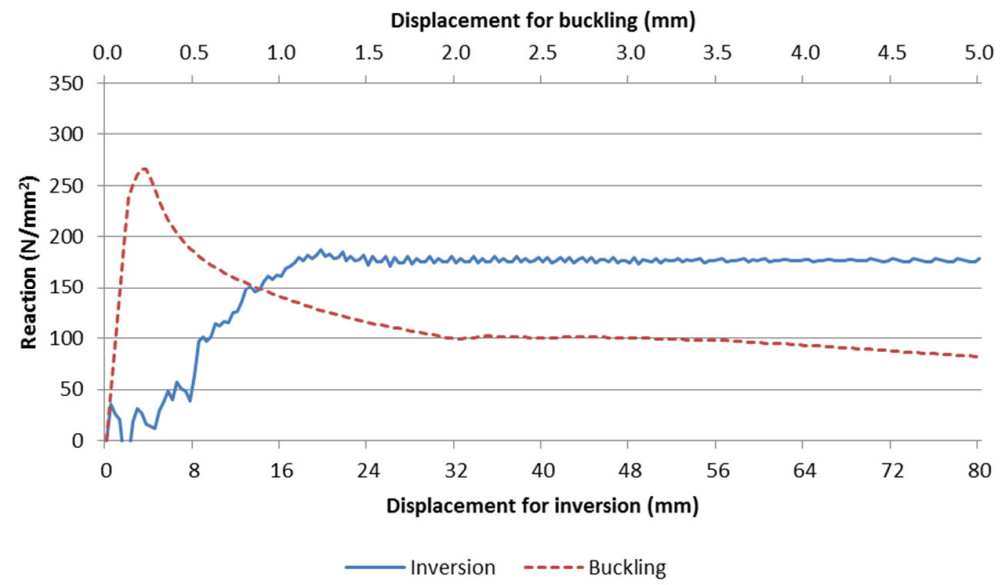

(c)

Figure 9. Reaction versus displacement curves of imperfect corrugated tubes buckling and inverting with (a) $0.5 \mathrm{~mm}$; (b) $0.35 \mathrm{~mm}$; and (c) $0.25 \mathrm{~mm}$ thickness. 


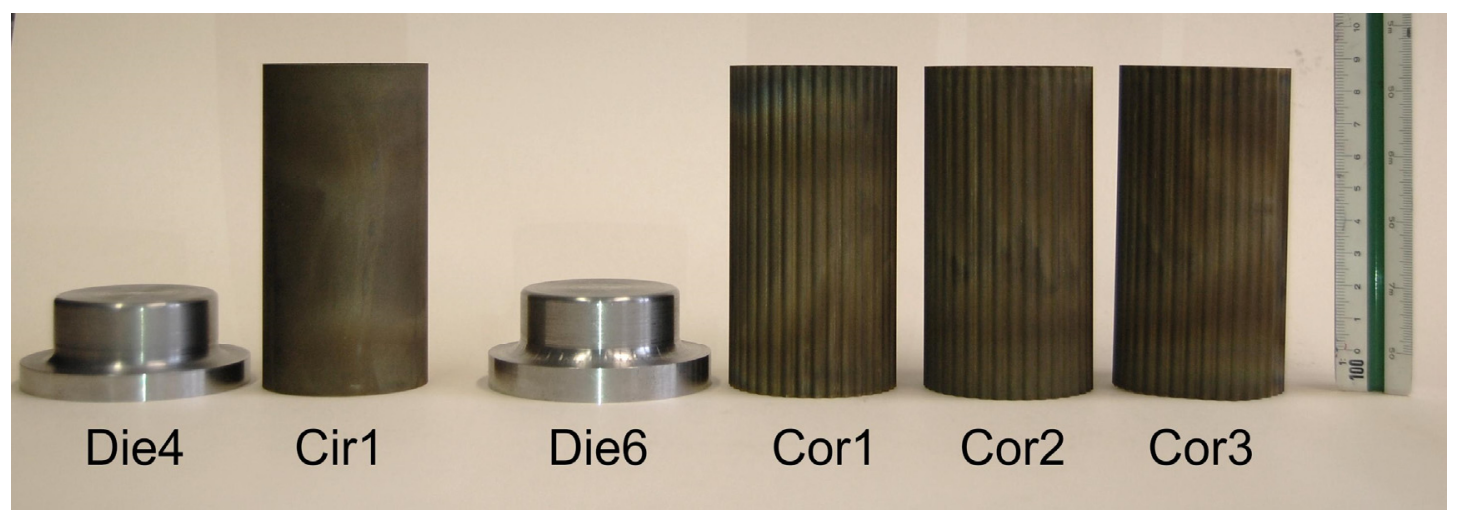

(a)
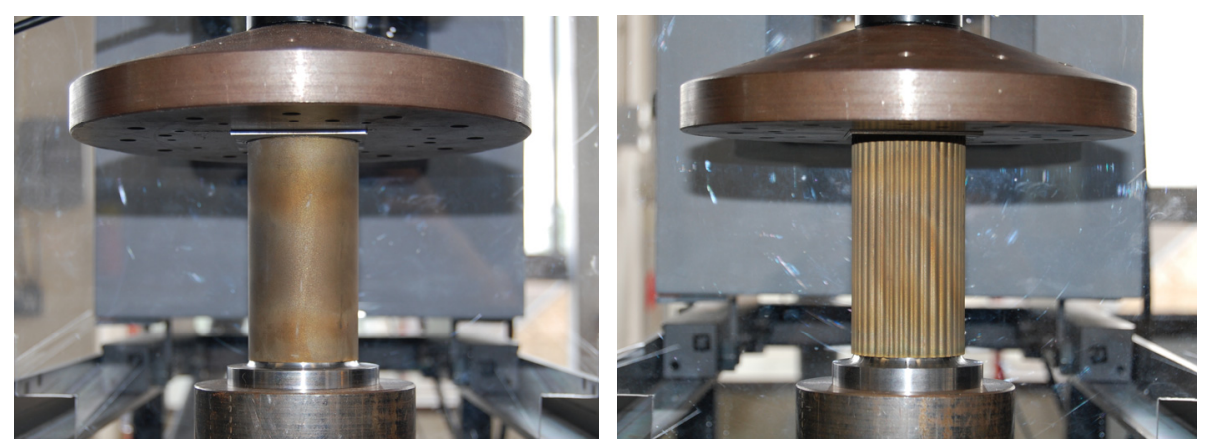

(b)

Figure 10. (a) Test specimens and dies; (b) compression test settings of for circular tube and corrugated tubes, respectively. 

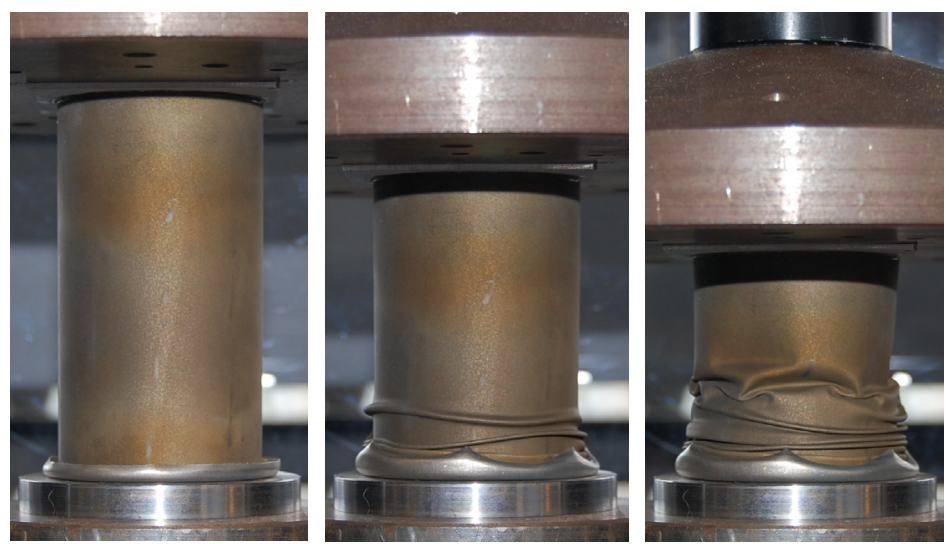

(a)
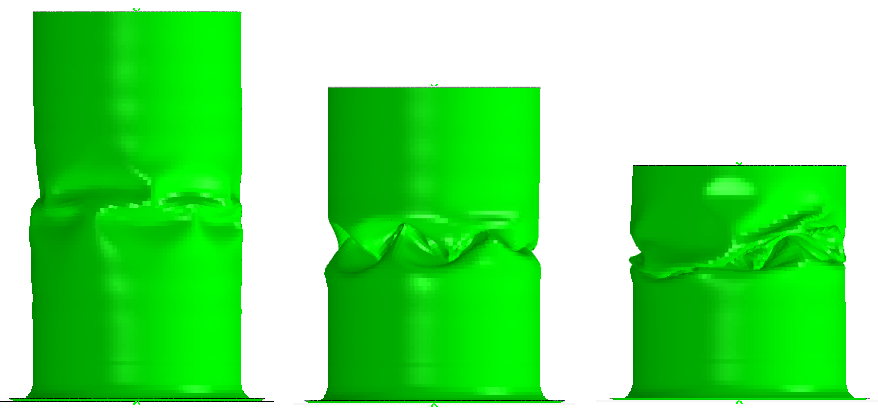

(b)

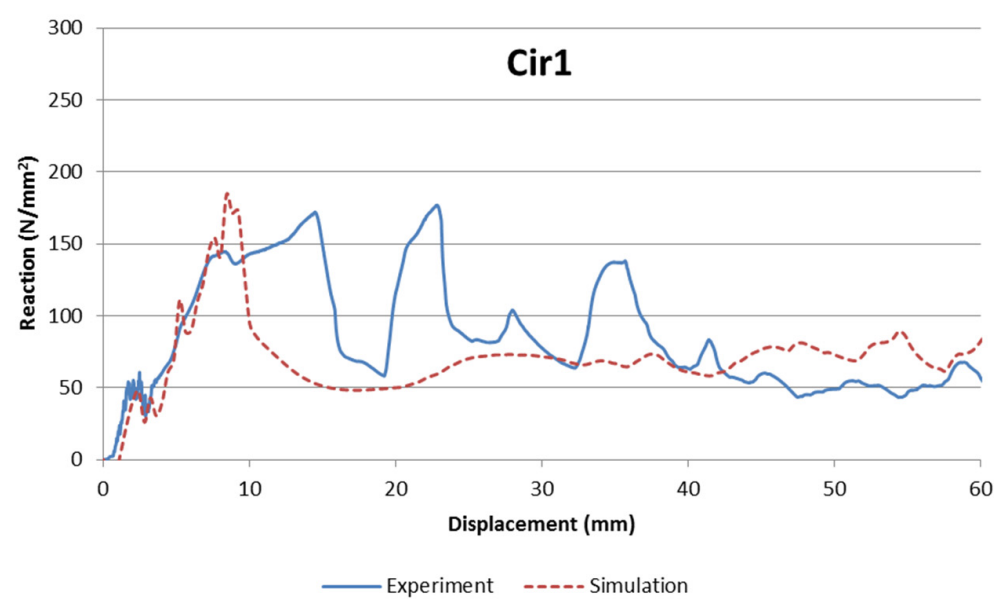

Figure 11. Experimental and numerical results of the circular tube Cir 1. (a) Experimental deformation with displacements of $10 \mathrm{~mm}, 30 \mathrm{~mm}$, and $50 \mathrm{~mm}$ respectively; (b) deformations from simulation for the tube at the same displacements; (c) reactions vs. displacements curves for both experiments and numerical simulation. 


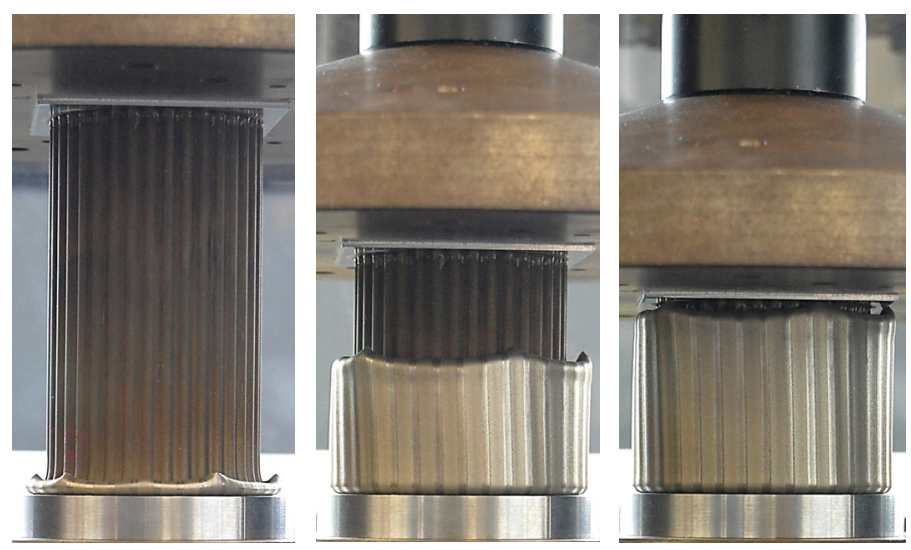

(a)
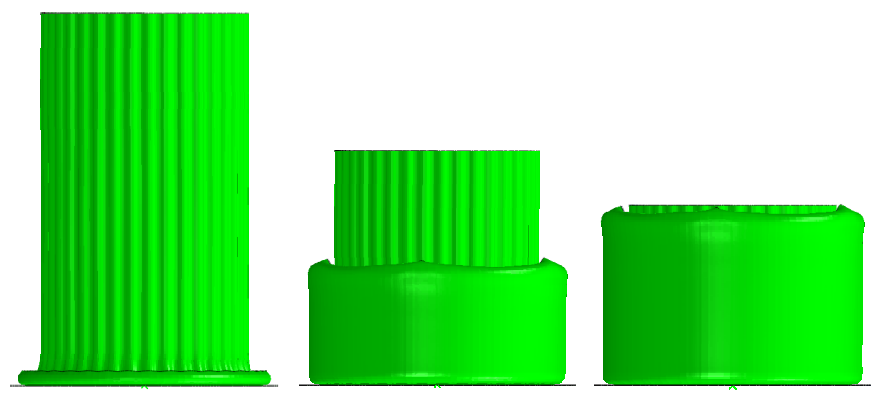

(b)

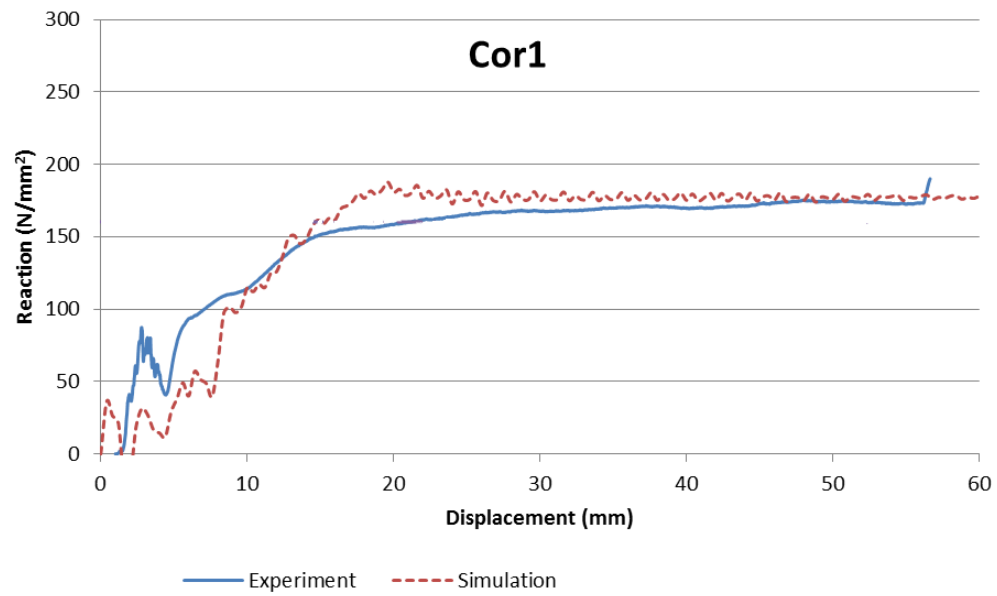

(c)

Figure 12. Experimental and numerical results of corrugated tube inversion with $t=0.25 \mathrm{~mm}$. (a) Experimental deformation of the tube with displacements of $13.3 \mathrm{~mm}, 46.7 \mathrm{~mm}$, and $60 \mathrm{~mm}$, respectively; the matching ones based on numerical simulation are shown in (b); (c) Reaction vs. displacement curves from both experimental and numerical results. 

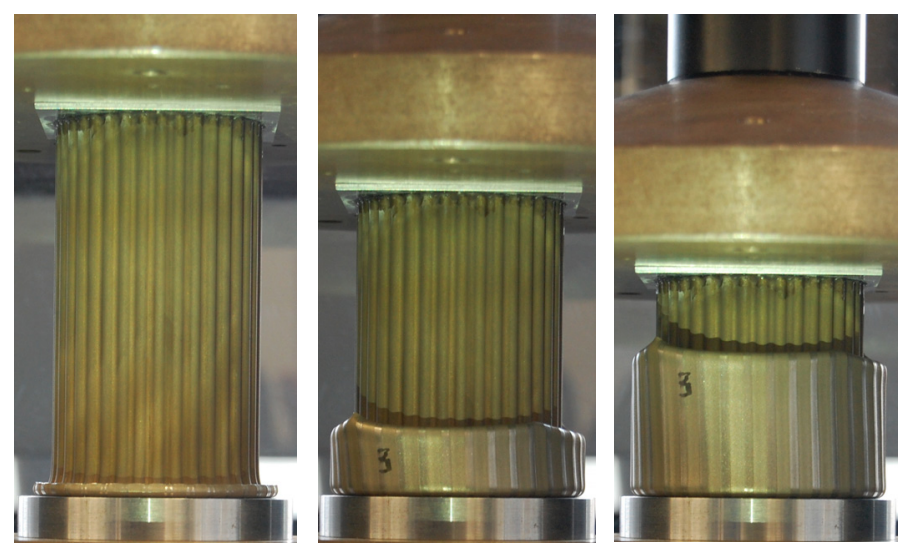

(a)
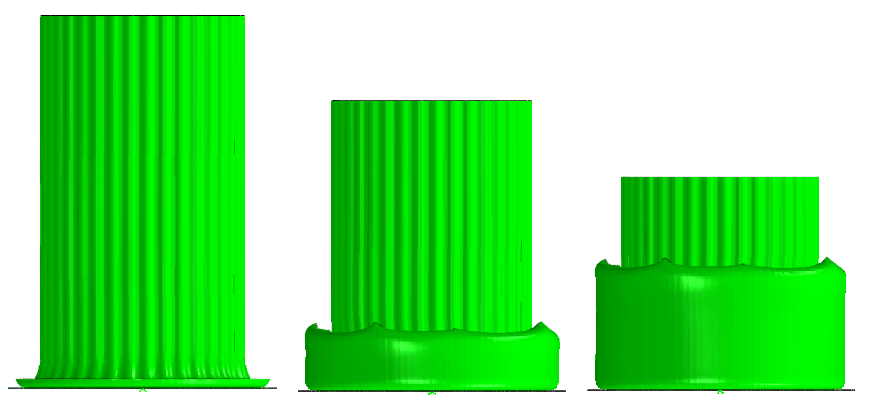

(b)

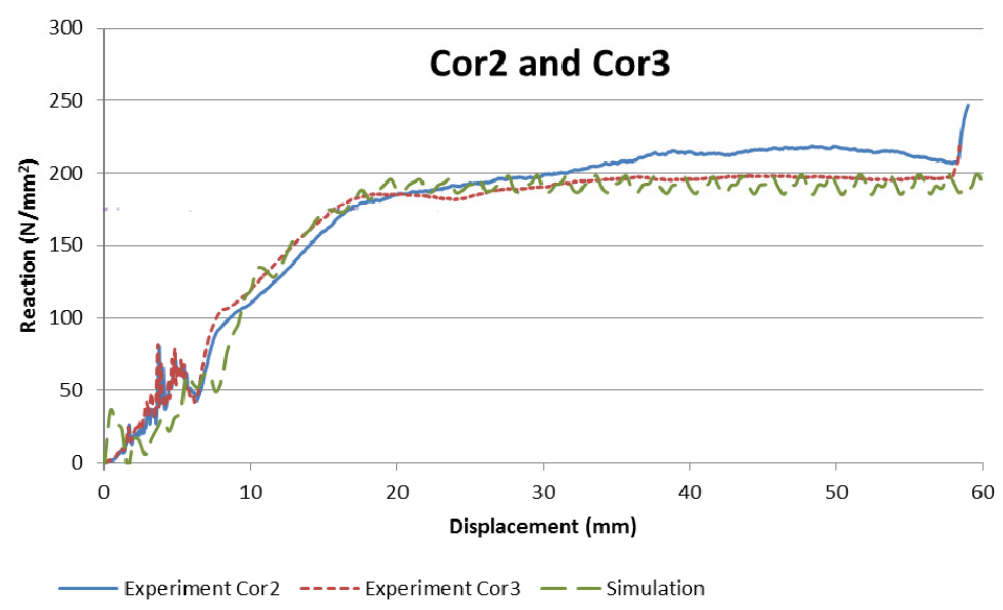

(c)

Figure 13. Experimental and numerical results of Cor 3 with $t=0.29 \mathrm{~mm}$. (a) Experimental deformation of the tube with displacements of $10 \mathrm{~mm}, 30 \mathrm{~mm}$, and $50 \mathrm{~mm}$, respectively; the matching ones based on numerical simulation are shown in (b); (c) Reaction vs. displacement curves from both experimental and numerical results. 


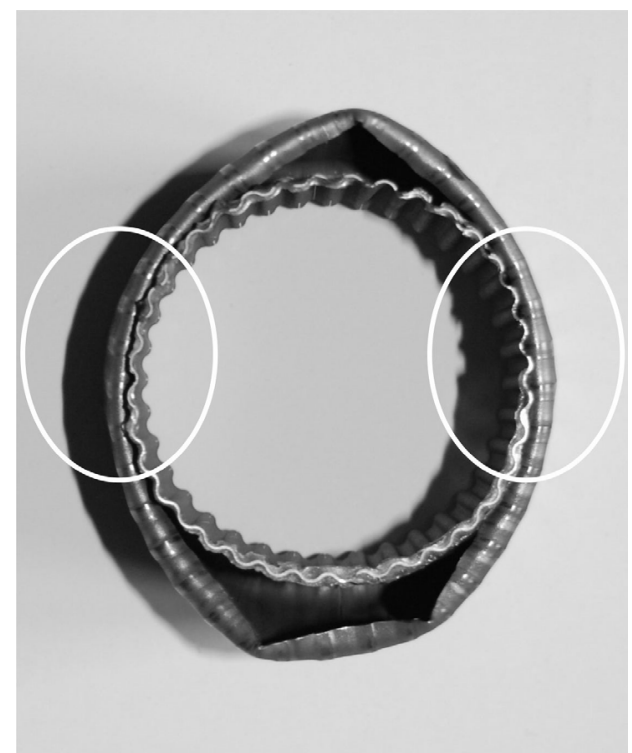

Figure 14. Self-contact of Cor2.

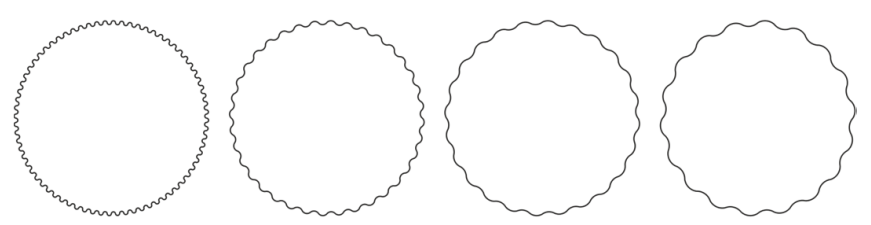

(a)

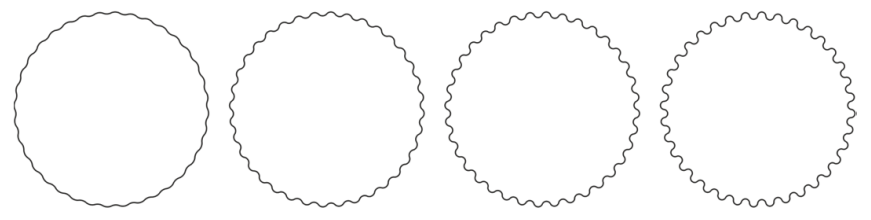

(b)

Figure 15. Cross-sectional shapes of corrugated tubes with different values of (a) $\theta$ and (b) $\alpha$. 